\title{
Inclusão da epistasia em modelo de avaliação genética de bovinos de corte compostos
}

[The effect of the inclusion of epistasis on the genetic evaluation model of beef cattle composite]

\author{
R.S. Bueno ${ }^{1}$, R.A. Torres ${ }^{2}$, J.B.S. Ferraz ${ }^{1}$, P.S. Lopes $^{2}$, J.P. Eler ${ }^{1}$, M. Almeida e Silva ${ }^{3}$, \\ R.F. Euclydes ${ }^{2}$, E.C. Mattos ${ }^{1}$
}

${ }^{1}$ Faculdade de Zootecnia e Engenharia de Alimentos - Universidade de São Paulo

Av. Duque de Caxias Norte, $\mathrm{n}^{\circ} 225$ - Campus da USP

13635-900 - Pirassununga, SP

${ }^{2}$ Universidade Federal de Viçosa - Viçosa, MG

${ }^{3}$ Universidade Federal de Minas Gerais - Belo Horizonte, MG

\begin{abstract}
RESUMO
Dados de bovinos compostos foram analisados para avaliar o efeito da epistasia nos modelos de avaliação genética. As características analisadas foram os pesos aos 205 (P205) e 390 dias (P390) e perímetro escrotal aos 390 dias (PE390). As análises foram realizadas pela metodologia de máxima verossimilhança considerando-se dois modelos: o modelo 1 incluiu como covariáveis os efeitos aditivos diretos e maternos, e os não aditivos das heterozigoses para os efeitos diretos e para o materno total, e o modelo 2 considerou também o efeito direto de epistasia. Para comparação dos modelos, foram utilizados o critério de informação de Akaike (AIC) e o critério de informação Bayesiano de Schwartz (BIC), e o teste de razão de verossimilhança. A inclusão da epistasia no modelo de avaliação genética pouco alterou as estimativas de componentes de (co)variâncias genéticas aditivas e, consequentemente, as herdabilidades. $\mathrm{O}$ teste de verossimilhança e o critério de Akaike sugeriram que o modelo 2, que inclui a epistasia, apresentou maior aderência aos dados para todas as características analisadas. O critério BIC indicou este modelo como o melhor apenas para P205. Para análise genética dessa população, o modelo que considerou o efeito de epistasia foi o mais adequado.
\end{abstract}

Palavras-chave: cruzados, efeitos não aditivos, perímetro escrotal, pesos

\begin{abstract}
Composite bovine data was analyzed with the objective of evaluating the effect of the epistasis parameter in the models of genetic evaluation. The analyzed characteristics were weight at 205 (W205) and 390 days (P390), and scrotal circumference at 390 days (SC390). The analysis were done by the maximum likelihood method, considering two models: model 1, which included as covariates the direct and maternal additive effects, and non-additive of the heterozygosis for the direct and total maternal, and model 2, which also considered the direct epistasis direct. The Akaike Information Criteria (AIC) and the Bayesiano of Schwartz Information Criteria (BIC) were used for the comparison of the models and the test of ratio of likelihood. The inclusion of the epistasis effects on the model of the genetic evaluation did not alter much the estimation of the genetic additive (co)variances components and, consequently the heritability. However, it was significantly superior by the likelihood ratio test for the studied characteristics. Through the BIC, model 2 was more adequate only for W205. For the genetic analysis of that population the model that considers the epitasis is the more adequate.
\end{abstract}

Keywords: crossbreed, non-additive genetic effects, scrotal circumference, weights

Recebido em 20 de julho de 2010

Aceito em 9 de junho de 2011

E-mail: rbueno@usp.br 


\section{INTRODUÇÃ̃O}

Aumento considerável na produção de bovinos de corte por meio do melhoramento genético tem sido alcançado por seleção, cruzamento e pela combinação de ambos, entretanto os modelos usados em avaliações genéticas e programas de melhoramento de animais cruzados e compostos consideram apenas efeitos aditivos e de dominância. Justificativa para o uso de modelos aditivo-dominante a fim de medir o desempenho esperado em populações de animais cruzados baseia-se na pressuposição de que a heterose é principalmente atribuída aos efeitos de dominância.

Estudos desenvolvidos por Gregory et al. (1991) sugerem que a retenção de heterose é linearmente proporcional à heterozigose. Dickerson (1973) propôs a utilização do termo "perdas por recombinação", que seriam proporcionais aos desvios de linearidade entre a heterose e a heterozigose.

A relação de proporcionalidade linear entre heterose e heterozigose também foi observada por Arthur et al. (1999) e Fries et al. (2000). No entanto, esses autores recomendaram que a adição dos efeitos de epistasia aos programas de avaliação genética pode melhorar as estimativas dos valores genéticos dos dados multirraciais ou de cruzamentos e que os efeitos epistáticos são importantes na constituição do genótipo animal.

Apesar de estarem fortemente associados aos componentes aditivos e de dominância, o que dificulta sua estimação, existem evidências de efeitos epistáticos sobre características de produção em cruzamentos de Bos indicus e Bos taurus. Este fato justifica a sistemática adição e testes de componentes epistáticos aos modelos operacionais, para populações multirraciais ou oriundas de cruzamentos avançados.

O objetivo deste trabalho foi avaliar a inclusão do efeito de epistasia em modelos de avaliação genética para características de peso e perímetro escrotal em uma população de bovinos de corte compostos.

\section{MATERIAL E MÉTODOS}

Foram analisados dados de peso ao desmame (P205) e aos 390 dias (P390) e do perímetro escrotal aos 390 dias (CE390) de bovinos compostos Montana Tropical ${ }^{\circledR}$, relativos a animais nascidos no período de 1994 a 2004. Os animais foram agrupados pela composição em tipos biológicos, N (Bos indicus, zebuínos), A (Bos taurus, taurinos adaptados às condições tropicais), B (Bos taurus, taurinos de origem britânica) e C (Bos taurus, taurinos de origem continental), segundo suas semelhanças de tipo, função, fisiologia, aspectos de crescimento e reprodução.

Os dados foram analisados, por meio da metodologia da máxima verossimilhança, utilizando-se o procedimento MIXED do software SAS (SAS, 1999). Dois modelos que diferiam quanto aos efeitos genéticos foram empregados: o modelo 1 incluiu como covariáveis os efeitos aditivos diretos e maternos, e os não aditivos das heterozigoses para os efeitos diretos e para o materno total, e o modelo 2, que considerou todos os efeitos do modelo acrescido do efeito de epistasia direto. Os efeitos ambientais incluídos nos modelos foram grupo contemporâneo e classe de idade da mãe ao parto. Os grupos de contemporâneos foram definidos como animais de mesmo sexo, nascidos na mesma safra e fazenda, e criados no mesmo grupo de manejo.

Os efeitos de heterose direta e materna foram obtidos a partir das heterozigoses diretas, calculadas segundo a fórmula, $h_{i x k}=\sum_{i \neq j} r_{i} r_{j}$, sendo $r$ a porcentagem de contribuição do tipo biológico baseado no sistema NABC para pai i e mãe $\mathrm{j}$.

As heterozigoses diretas foram consideradas: $\mathrm{NxA}=$ porcentagem de cruzamento de raças do tipo biológico $\mathrm{N}$ e A existente na composição racial do animal; $\mathrm{NxB}=$ porcentagem de cruzamento de raças do tipo biológico $\mathrm{N}$ e $\mathrm{B}$ existente na composição racial do animal; $\mathrm{NxC}$ = porcentagem de cruzamento de raças do tipo biológico $\mathrm{N}$ e $\mathrm{C}$ existente na composição racial do animal; $\mathrm{AxB}=$ porcentagem de cruzamento de raças do tipo biológico $\mathrm{A}$ e $\mathrm{B}$ existente na composição racial do animal; $\mathrm{AxC}=$ porcentagem de cruzamento de raças do tipo biológico A e $\mathrm{C}$ existente na composição racial do animal; $\mathrm{BxC}=$ porcentagem do cruzamento de raças do tipo biológico $\mathrm{B}$ e $\mathrm{C}$ existente na 
composição racial do animal. A heterozigose materna total (MT) foi expressa como a soma das heterozigoses parciais, como obtida acima, porém foi calculado um valor total para a mãe do animal.

Quanto ao efeito de epistasia, foi incluso o coeficiente proposto por Dickerson (1973), segundo Koch (1985), $r^{i}=2\left(1-\sum f i^{2}\right)-h z^{i}$, em que $f i$ é a proporção de cada raça no individuo, e $h z^{i}$ heterozigose do indivíduo.

A comparação dos modelos foi feita pelo critério de informação de Akaike (AIC), ou seja, critério de informação Bayesiano de Schwartz (BIC), e o teste de razão de verossimilhança foi aplicado aos modelos.

A estimativa da estatística do teste da razão de verossimilhança (LR) (Rao, 1973) foi comparada com o valor obtido pela distribuição de quiquadrado $\left(\chi^{2}\right)$, com 1 grau de liberdade e nível de significância fixo de 5\%. Para AIC e BIC, o valor para comparação foi obtido por: $\mathrm{AIC}=-$ $2 \ln L+2 p$ e $\mathrm{BIC}=-2 \ln L+p \ln (\mathrm{N}-\mathrm{r})$, em que: $\mathrm{p}$ refere-se ao número de parâmetros do modelo; $\mathrm{N}$, ao número total de informações; e $\mathrm{r}$, ao posto da matriz X, matriz de incidência dos efeitos fixos. Menores valores de AIC e BIC indicam modelos mais adequados.

Os dados foram submetidos ao pré-ajuste para os efeitos não aditivos estimados, conforme recomendações de Mourão (2005). Estimativas de componentes de (co)variâncias e de parâmetros genéticos foram obtidas pelo procedimento de máxima verossimilhança restrita usando um algoritmo livre de derivadas, sob um modelo animal que considerou como efeitos aleatórios, aditivo direto e materno e residual e para P205, também o efeito permanente de meio materno, utilizando-se o programa MTDFREML (Boldman et al., 1995), que foram comparadas com as análises univariadas para as características em estudo realizadas por Mourão (2005).

\section{RESULTADOS E DISCUSSÃO}

A estatística descritiva dos dados do P205, P390 e PE390 é apresentada na Tab. 1. Médias, desvio-padrão e coeficientes de variação das características são similares às reportadas na literatura (Eler et al., 2000, Mourão, 2007).

Tabela 1. Número de observações (N), média geral, desvio-padrão (DP) e coeficiente de variação obtidos para as características em estudo

\begin{tabular}{lrrr}
\hline Parâmetro & P205 $(\mathrm{kg})$ & $\mathrm{P} 390(\mathrm{~kg})$ & PE390 $(\mathrm{cm})$ \\
\hline N & 118.266 & 58.006 & 25.849 \\
Média & 191,59 & 272,68 & 28,28 \\
DP & 31,43 & 50,07 & 3,86 \\
CV $(\%)$ & 11,52 & 9,46 & 10,52 \\
\hline
\end{tabular}

Os valores do logaritmo natural da função de verossimilhança $(\mathrm{Ln} L)$ para os modelos, em cada característica, os valores da razão de verossimilhança (LR) e dos critérios de informação de Akaike (AIC) e Bayesiano (BIC) são apresentados na Tab. 2.

A inclusão do efeito de epistasia no modelo provocou reduções significativas para P205 $(\mathrm{P}<0,01), \mathrm{P} 390$ e CE390 $(\mathrm{P}<0,05)$ no logaritmo natural da função de verossimilhança, conforme teste da razão de verossimilhança que indica superioridade do modelo 2 em relação ao 1 . Demeke et al. (2003b), ao testarem modelos diferentes quanto à quantificação dos efeitos não aditivos, também verificaram que os modelos que consideraram os efeitos epistáticos apresentaram maior aderência aos dados em relação aos modelos de dominância. Entre os modelos epistáticos, o modelo de Dickerson apresentou melhor $\mathrm{R}^{2}$ em relação aos demais.

Kahi et al. (2000) compararam quatro modelos genéticos: modelo dominante, modelo que consideraram os efeitos de recombinação (ou epistasia), Dickerson e Kinghorn, e um modelo que considerou os efeitos de dominância e interações aditiva $\mathrm{x}$ aditiva, na estimação de efeitos aditivos e não aditivos de uma população de bovinos compostos do Kenya. O modelo de Dickerson foi o mais adequado para analisar a referida população. 
Tabela 2. Valores do logaritmo natural das funções de verossimilhança $(\operatorname{Ln} L)$, critério de informação de Akaike (AIC), critério de informação Bayesiano de Schwartz (BIC) para modelos sem e com a inclusão do efeito de epistasia, e teste da razão de verossimilhança (LR)

\begin{tabular}{ccccccc}
\hline \multicolumn{2}{c}{ P205 } & \multicolumn{2}{c}{ P390 } & \multicolumn{2}{c}{ CE390 } \\
\hline & 1 & 2 & 1 & 2 & 1 & 2 \\
\hline LnL & -532.118 & -532.092 & $-268.771,3$ & $-268.769,4$ & $-63.899,9$ & $-63.897,7$ \\
& LR = 52 & $(\mathrm{P}<0.01)^{1}$ & LR $=3,8$ & $(\mathrm{P}<0.05)$ & LR $=4,4(\mathrm{P}<0.05)$ \\
\hline AIC & 1.071 .016 & 1.070 .966 & $545.628,6$ & $545.626,8$ & $131.523,8$ & $131.521,4$ \\
\hline BIC & 1.103 .834 & 1.103 .793 & $581.887,4$ & $581.894,6$ & $146.717,8$ & $146.723,6$ \\
\hline
\end{tabular}

${ }^{1}$ LR para modelo sem e com epistasia.

Para todas as características em estudo, a covariância residual estimada pelos modelos foi menor no modelo 2 (informação não apresentada).

Segundo os critérios de AIC e BIC, um modelo é mais apropriado quanto menor são essas estatísticas. Assim, pelo AIC, o modelo que proporcionou melhor aderência aos dados foi o que incluiu o efeito de epistasia (modelo 2), para todas as características. No entanto, pelo BIC, o modelo 2 apresentou maior aderência somente para P205. Vale ressaltar que ambos, AIC e BIC, penalizam modelos com maior número de parâmetros. Entretanto, para BIC, esta penalidade é mais rigorosa e favorece modelos mais parcimoniosos (Nunez-Antón e Zimmerman, 2000).

Os componentes de (co)variâncias e parâmetros genéticos estimados quando os dados foram ajustados para os efeitos não aditivos dos modelos 1 (heterozigoses diretas e heterozigose materna total) e 2 (heterozigoses diretas, heterozigose materna total e epistasia direta) estão na Tab. 3 .

Ao se comparar os modelos, as estimativas de componentes de (co)variâncias tiveram comportamento similar, e as magnitudes não diferiram.

Tabela 3. Estimativas de componentes de (co)variância e parâmetros genéticos obtidos a partir dos modelos 1 e 2, para as características peso aos 205 e 390 dias, e perímetro escrotal aos 390 dias ajustadas para os efeitos não aditivos

\begin{tabular}{|c|c|c|c|c|c|c|c|c|c|c|c|}
\hline \multirow[b]{3}{*}{ Modelo } & \multicolumn{6}{|c|}{ Componente de (co)variância $^{1}$} & \multicolumn{5}{|c|}{ Parâmetros genéticos } \\
\hline & \multicolumn{11}{|c|}{ Peso aos 205 dias (P205) } \\
\hline & $\sigma_{a}^{2}$ & $\sigma_{a m}$ & $\sigma_{m}^{2}$ & $\sigma_{c}^{2}$ & $\sigma_{e}^{2}$ & $\sigma_{p}^{2}$ & $\hat{h}_{a}^{2}$ & $\hat{r}_{a m}$ & $\hat{h}_{m}^{2}$ & $\hat{c}$ & $\hat{e}^{2}$ \\
\hline 1 & 139,614 & $-70,257$ & 119,163 & 36,714 & 302,450 & 527,685 & 0,26 & $-0,54$ & 0,23 & 0,070 & 0,57 \\
\hline \multirow[t]{2}{*}{2} & 130,200 & $-63,798$ & 114,172 & 37,511 & 307,207 & 525,291 & 0,25 & $-0,52$ & 0,22 & 0,071 & 0,58 \\
\hline & \multicolumn{11}{|c|}{ Peso aos 390 dias (P390) } \\
\hline 1 & 153,251 & $-32,941$ & 82,733 & & 505,131 & 708,174 & 0,22 & $-0,29$ & 0,12 & & 0,71 \\
\hline \multirow[t]{2}{*}{2} & 151,386 & $-27,534$ & 77,566 & & 506,337 & 707,756 & 0,21 & $-0,25$ & 0,11 & & 0,72 \\
\hline & \multicolumn{11}{|c|}{ Perímetro escrotal aos 390 dias (PE390) } \\
\hline 1 & 1,561 & $-0,223$ & 0,701 & & 7,119 & 9,157 & 0,17 & $-0,21$ & 0,08 & & 0,78 \\
\hline 2 & 1,570 & $-0,306$ & 0,773 & & 7,124 & 9,161 & 0,17 & $-0,28$ & 0,08 & & 0,78 \\
\hline
\end{tabular}

${ }^{1} \sigma_{a}^{2}=$ variância genética aditiva direta; $\sigma_{a m}=$ covariância genética direta e materna; $\sigma_{m}^{2}=$ variância genética aditiva materna, $\sigma_{c}^{2}=$ variância do efeito permanente de meio; $\sigma_{e}^{2}=$ variância residual; $\sigma_{p}^{2}=$ variância fenotípica; $\hat{h}_{a}^{2}=$ herdabilidade direta; $\hat{r}_{a m}=$ correlação aditiva-materna; $\hat{h}_{m}^{2}=$ herdabilidade materna; $\hat{c}$ =proporção do efeito permanente de meio; $\hat{e}^{2}=$ proporção do efeito residual.

Para P205 e P390, os componentes de variâncias genéticas, aditiva direta e materna foram menores quando estimadas pelo modelo 2 , e a covariância genética direta e materna foi negativa para todos os modelos e características em estudo. 
As diferenças observadas para os componentes de (co)variância foram pequenas, constatadas nas estimativas de herdabilidade que diferiram em 0,01 para P205 e P390, e não diferiram para PE390 entre os modelos.

A estimativas de herdabilidade direta obtidas para P205 foram 0,26 e 0,25, e materna 0,23 e 0,22 , respectivamente, para os modelos 1 e 2 . O componente de variância para o efeito permanente de meio foi 0,07 para ambos os modelos. Para P390, as herdabilidades diretas foram 0,22 e 0,21 , e maternas de 0,12 e 0,11 , para os modelos 1 e 2 , respectivamente.

As estimativas de herdabilidades direta encontradas neste estudo para características de pesos foram maiores que as encontradas por Demeke et al. (2003a) para uma população multirracial na Etiópia, os quais obtiveram estimativas de 0,09 e 0,12, para os pesos a desmama e aos 12 meses. Gregory et al. (1995) relataram maiores herdabilidades, com estimativas de 0,21 e 0,38 para peso aos 200 dias; de 0,31 e 0,43 para peso aos 368 dias, e de 0,24 e 0,40 para peso aos 410 dias, em animais compostos e cruzados, respectivamente.

Estimativas de herdabilidades semelhantes foram obtidas por Mourão (2005), ao trabalhar com a mesma população de bovinos compostos.

Em geral, as estimativas encontradas neste estudo estão dentro da variação apresentada na literatura para as características.

\section{CONCLUSÕES}

O uso de modelos mais simples para avaliação de bovinos compostos pode erroneamente identificar a contribuição dos efeitos aditivos e não aditivos como efeito de heterose. Modelos que incluam os efeitos de epistasia são mais adequados para análise de populações multirraciais.

\section{AGRADECIMENTOS}

À Agropecuária CFM Ltda., pelo fornecimento dos dados, e ao CNPq, pelo apoio financeiro.

\section{REFERÊNCIAS BIBLIOGRÁFICAS}

ARTHUR, P.F.; HEARSHAW, H.; STEPHENSON, P.D. Direct and maternal additive and heterosis effect from crossing Bos indicus and Bos taurus cattle: Cow and calf performance in two environments. Livest. Prod. Sci., v.57, p.231-241, 1999.

BOLDMAN, K.G.; KRIESE, L.A.; VAN VLECK, L.D. et al. A manual for use of MTDFREML: a set of programs to obtain estimates of variances and covariances (DRAFT). Lincoln: Department of Agriculture/Agriculture Research Service, 1995. $125 \mathrm{p}$.

DEMEKE, S.; NESER, F.W.C.; SCHOEMAN, S.J. Early growth performance of Bos taurus $\times$ Bos indicus cattle crosses in Ethiopia: Evaluation of different crossbreeding models. J. Anim. Breed. Gen., v.120, p.39-50, 2003a.

DEMEKE, S.; NESER, F.W.C.; SCHOEMAN, S.J. Variance components and genetic parameters for early growth traits in a mixed population of purebred Bos indicus and crossbred cattle. Livest. Prod. Sci., v.84, p.11-21, 2003b.

DICKERSON, G.E. Inbreeding and heterosis in animals. In: Proceedings of the Animal Breeding and Genetics Symposium. Am. Soc. Anim. Sci., p.54-77, 1973.

ELER, J.P.; FERRAZ, J.B.S.; GOLDEN, B.L. et al. Influência da interação touro $\mathrm{x}$ rebanho na estimação da correlação entre efeitos genéticos direto e materno em bovinos da raça Nelore. Rev. Bras. Zootec., v.29, p.1642-1648, 2000.

FRIES, L.A.; JOHNSTON, D.J.; HEARNSHAW, H. et al. Evidence of epistatic effects on weaning weight in crossbreed beef cattle. Asian-Aust. J. Anim. Sci., v.13, suppl. B, p.242, 2000.

GREGORY, K.E.; LUNSTRA, D.D.; CUNDIFF, L.V. et al. Breed effects and heterosis in advanced generations of composite populations for puberty and scrotal traits of beef cattle. $J$. Anim. Sci., v.69, p.2795-2807, 1991.

GREGORY, K.E.; CUNDIFF, L.V.; KOCH, R.M. Genetic and phenotypic (co)variances for growth and carcass traits of purebred and composite populations of beef cattle. J. Anim. Scie., v.73, p.1920-1926, 1995. 
KAHI, A.K.; THORPE, W.; NITTER, G. et al. Crossbreeding for dairy production in the lowland tropics of Kenya: I. Estimation of individual crossbreeding effects on milk production reproductive traits and on cow live weight. Livest. Prod. Sci., v.63, p.39-54, 2000.

MOURÃO, G.B.; FERRAZ, J.B.S.; ELER, J.P. et al. Genetic parameters for growth traits of a Brazilian Bos taurus $\mathrm{x}$ Bos indicus beef composite. Genet. Mol. Res., v.6, p.1190-1200, 2007.

MOURAO, G.B. Estimação de efeitos genéticos aditivos diretos e maternos e não aditivos $e$ predição do desempenho de pesos, perímetro escrotal e musculosidade em uma população de bovinos de corte compostos (Bos taurus x Bos indicus). 2005. 109f. Tese (Doutorado em Zootecnia) - Faculdade de Zootecnia e Engenharia de Alimentos/ Universidade de São Paulo, Pirassununga, SP, 2005.
NUNEZ-ANTÓN, V.N.; ZIMMERMAN, D.L. Modelling nonstationary longitudinal data. Biometrics., v.56, p.699-705, 2000.

RAO, C.R. Linear statistical inference and its aplications. 2.ed. New York: John Wiley \& Sons. 1973. p.417-420.

STATISTICAL ANALYSES SYSTEM - SAS. SAS/STAT user'guide, version 8.0 ed. Cary: 1999. v.1, 943p. 